\title{
Estimation of Inherent Governor Dead-Band and Regulation Using Unscented Kalman Filter
}

\author{
Pratyasa Bhui, Student Member, IEEE, Nilanjan Senroy, Member, IEEE, Abhinav K. Singh, Member, IEEE, \\ and Bikash C. Pal, Fellow, IEEE
}

\begin{abstract}
The inclusion of the governor droop and dead-band in dynamic models helps to reproduce the measured frequency response accurately and is a key aspect of model validation. Often, accurate and detailed turbine-governor information are not available for various units in an area control centre. The uncertainty in the droop also arise from the nonlinearity due to the governor valves. The droop and deadband are required to tune the secondary frequency bias factors, and to determine the primary frequency reserve. Earlier research on droop estimation did not adequately take into account the effect of dead-band and other nonlinearities. In this paper, unscented Kalman filter is used in conjunction with continuously available measurements to estimate the governor droop and the dead-band width. The effectiveness of the approach is demonstrated through simulations.
\end{abstract}

Index Terms-Load frequency control, parameter estimation, unscented Kalman filter, primary frequency response, governor dead-band.

\section{NOMENCLATURE}

Subscript $k k^{\text {th }}$ time instant.

$\Delta \quad$ Deviation from the rated value.

$E($.$) \quad Expected value of ".".$

". ." Estimated value ".".

$R \quad$ Droop constant.

$K_{p} \quad$ Gain of the power system transfer function.

$T_{p} \quad$ Time Constant of the power system TF

$\beta \quad$ Area frequency response characteristics

$B \quad$ Secondary frequency bias $(M W / 0.1 H z)$

$A C E \quad$ Area Control Error

$\Delta P_{t i e} \quad$ Tie line power deviation

$K_{i} \quad$ Integral control constant

pf participation factor

$d \quad$ Governor deadband

$F_{h p} \quad$ Percentage of power through HP Turbine

$\Delta P_{m} \quad$ Deviation in Mechanical Power

$\Delta P_{\text {load }} \quad$ Deviation in Load Power

$u_{d k} \quad$ Input to the governor deadband at time $=k$

$y_{d k} \quad$ Output of the governor deadband at time $=k$

$x_{d 0} \quad$ Midposition of the governor deadband

This work was supported in part by the Department of Science and Technology, Government of India, and Research Council UK under its grant DST/RCUK/SEGES/2012/02 and EP/K036173/1.

Pratyasa Bhui and Nilanjan Senroy are with Dept of Electrical Engineering, Indian Institute of Technology Delhi, India (e-mail: nsenroy@ee.iitd.ac.in, pratyasansec@gmail.com).

Abhinav Kr. Singh is with the School of Engineering, University of Lincoln, UK, and Bikash C. Pal is with the Dept of Electrical and Electronic Engineering, Imperial College London, UK (email: asingh@lincoln.ac.uk, b.pal@imperial.ac.uk)

$\begin{array}{ll}P_{r e f} & \text { Reference Power } \\ \Delta P_{v} & \text { Deviation in Governor valve opening } \\ T_{g} & \text { Governor time constant } \\ \Delta P_{t} & \text { Deviation in turbine power } \\ T_{t} & \text { Turbine time constant } \\ \Delta P_{r} & \text { Deviation in power through reheater } \\ T_{r} & \text { Reheater Time Constant } \\ P_{e} & \text { Electrical Power } \\ D & \text { Generator damping constant } \\ H & \text { Generator inertia } \\ \omega & \text { Generator speed } \\ \boldsymbol{x}, \boldsymbol{x}^{-} & \text {System state and predicted state vector } \\ \mathbf{u} & \text { System input vector } \\ \mathbf{q} & \text { Process noise } \\ \mathbf{Q} & \text { Process noise covariance matrix } \\ \mathbf{R} & \text { Measurement noise covariance matrix } \\ \mathbf{f} & \text { Nonlinear system model } \\ \mathbf{h} & \text { Measurement function } \\ \mathbf{P}, \mathbf{P}^{-} & \text {State and Predicted state covariance matrix } \\ \chi, \chi^{2} & \text { Sigma and predicated sigma points } \\ \mathbf{Y} & \text { Predicted measurement sigma points } \\ \boldsymbol{\mu} & \text { Predicted measurement vector } \\ \mathbf{y} & \text { Measurement } \\ \mathbf{S} & \text { Measurement covariance matrix } \\ \mathbf{C} & \text { Cross covariance of } \chi^{-} \text {and } Y^{-} \\ \mathbf{K} & \text { Kalman Gain matrix } \\ & \end{array}$

\section{INTRODUCTION}

I NVESTIGATIONS into the major blackouts in the world have revealed the inadequacy of updated dynamic models to simulate the actual system dynamics accurately. It has been noted in one study that more than 50\% plants in North America have inadequate information about turbine-governor time constants and other important parameters [1]. As a result, the simulated frequency response differs from the actual response following disturbances [2]. The primary frequency response (PFR) of the utilities may reduce because of diminishing inertia due to penetration of non-synchronous type generation into the system, lesser number of generators participating in frequency control, and ageing governor systems. It was concluded in [3] that existing standards may not be adequate in determining the reserve requirement and also revealed insufficient reserve and weak correlation between PFR and total load. Due to ancillary services pricing and uncertain system inertia, there is an increasing requirement of estimating the PFR of the system, optimizing the total reserve, as well as 
monitoring the reserve available from individual generators. In this context, online estimation of the parameters related to primary frequency control from measurements is crucial. Updated information about all these parameters can also be used in adaptive LFC, estimating PFR after a disturbance [4].

The speed and magnitude of the unit PFR is determined by the governor dead-band, ramp-rate constraints and the droop. The net droop of a control area is representative of its area frequency response characteristics $(\beta)$ and how stiff the area frequency is against load-generation unbalances. In practice, the actual $\beta$ of an area is seldom known, but it can be approximated from the overall area droop, and used as a frequency bias coefficient (stated as 10B, where $\mathrm{B}$ is in $\mathrm{MW} / 0.1 \mathrm{~Hz}$ ) in secondary frequency controllers [5]. Inaccurate setting of frequency bias causes frequency and tie line power oscillations impacting the overall dynamics [6]. Earlier research on estimation of $\beta$ was fairly accurate for generators without governor dead-band [7], [8], [9], [10], [11]. These methods, however, reported gross estimation errors with dead-band and other nonlinearities [7]. It was shown in [2] that inclusion of an intentional deadband in the governor simulation models improves the matching between the simulated and actual PFR. This is, however, not always a practical option. For instance, the Multiregional Modeling Working GroupTennessee Valley Authority (MMWG-TVA) model has 3000+ machines with a total capacity of $591 \mathrm{GW}$. Out of the 3000+ generators, 45.42 of the total generation capacity is attached with active governors and 92.07 of these generators have four basic types of governors- IEEEG1, IEEESGO, TGOV1 and GAST [2]. There is no scope for including unintentional governor deadband in these models and/as very often the information is unavailable [12].

In this paper, it is proposed to estimate the droop and governor dead-band width using the unscented Kalman filter (UKF). The method relies on three types of measurements the integral area control error (ACE) signal sent from the control centre, frequency measurements obtained from phasor measurement unit (PMU), and individual unit mechanical power. The mechanical power is not directly observable and may be obtained using the electrical power measurements and the accelerating power (which is calculated using the speed signal). The choice of UKF is guided by its superior ability to handle nonlinearities efficiently [13], when compared to other parameter estimation techniques used with power system measurements, such as least square [14], extended Kalman filter (EKF) [15], particle filter [16], trajectory sensitivity [17].

The rest of the paper is organized as follows the relevant mathematical models of load frequency control and UKF are briefly explained in Sec II and Sec III respectively. The performance of the method has been assessed in Sec IV using linear models and in Sec V for non-linear models. The performance of the proposed method has been compared with the recursive least square technique in Sec VI. The impact of measurement noise has been studied in Sec VII. Impact of GRC and bad data detection have been provided in Sec VIII and Sec IX respectively. Section X is the concluding section.

\section{MATHEMATICAL MODEL OF LOAD FREQUENCY CONTROL}

The linear transfer function model of a generator in LFC model, has been shown in Fig.1. The proposed method for estimating the droop and dead-band needs the measurements of the integral of the ACE i.e. $\int A C E$, electrical power and speed. Typically, the ACE signal may be distributed among multiple units in an area, based on participation factors. As the local frequencies may not be same even in the same control area, the local generator frequency should be used for parameter estimation of the corresponding generator. In this work, only one unit has been considered $(p f=1)$, however multiple units may be considered with $p f<1$ without any changes in the proposed method. The $\int A C E$ and the frequency deviation have been taken as inputs and the mechanical power as measured output in this estimation problem. As the mechanical power may not be directly measurable, it is calculated from the electrical power output and speed. The accelerating power, obtained from the speed measurement, is added with electrical power to get the mechanical power.

\section{A. Modeling of governor dead-band}

The governor dead-band is due to the backlash in the linkage between servo piston and the camshaft. It occurs in the rack and pinion used to rotate the camshaft operating the control valves. A describing function may be used to represent the dead-band, for studying step changes in load or generation [18]. However, such describing functions may not be accurate to study ambient continuous load changes, as the describing function output depends on the magnitude and frequency of the sinusoid input. Therefore, the governor dead-band has been represented here as a backlash function. The difficulty in estimating the dead-band width is that it is highly nonlinear and its output depends not only on the present input but also on the past output. Two extra variables were introduced-past output and the mid-position of the backlash, denoted as $y_{d(k-1)}$ and $x_{d 0}$ respectively. The width of the deadband/backlash is $d$, input and output of the deadband at $k^{t h}$ instant are $u_{d k}$ and $y_{d k}$, respectively. The mid-position of the dead-band is represented as $x_{d 0}$ (Fig.1.b ).

\section{B. Dead-band width}

Generally, the governor deadband is represented as the total magnitude of sustained frequency change within which there should be no resulting change in the valve position. The limiting value of dead-band is specified as $0.06 \%$ [11], [18]. If the droop $(R)$ of the generator is $2.4 \mathrm{~Hz} / \mathrm{puMW}$ and deadband width is $0.06 \%$, the governor dead-band width in terms of power is $\frac{\Delta f}{R}=(0.0006 * 60) / 2.4=0.015 p u M W$

\section{Mathematical model of the turbine-governor dynamics}

The aim of this work is to estimate the droop and the governor dead-band, and therefore the focus is on the turbinegovernor dynamics only. The input to the governor dead-band at $k^{t h}$ instant is given as follows.

$$
u_{d k}=\Delta P_{r e f}(k)-p f . \int A C E(k)-\frac{\Delta f(k)}{R}
$$




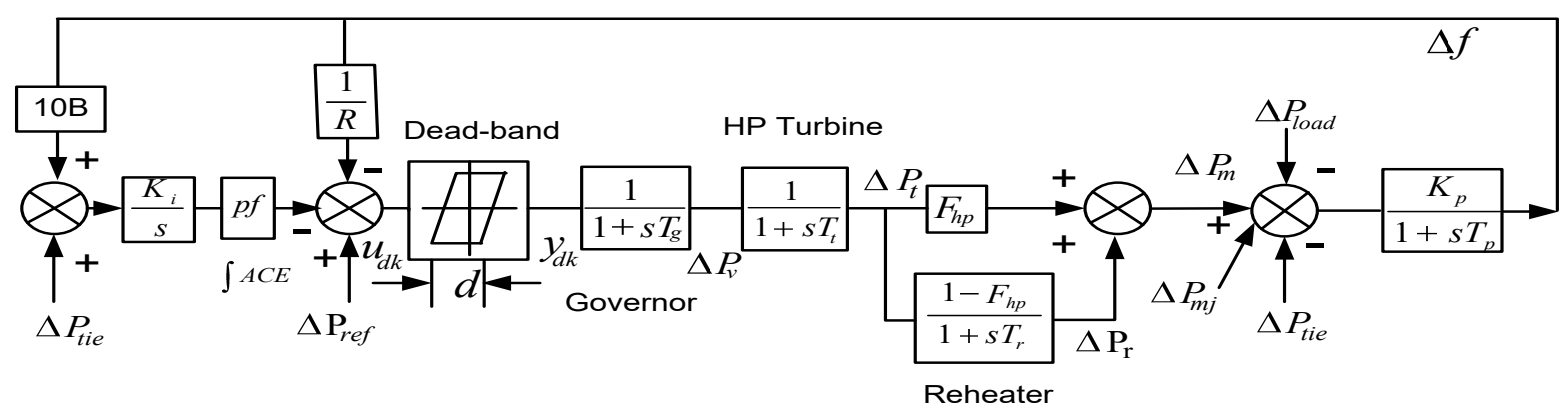

(a)

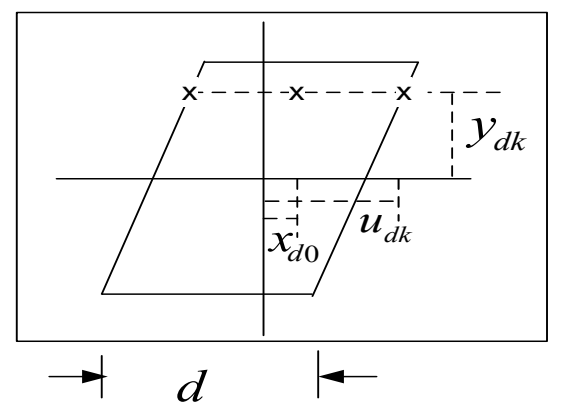

(b)

Fig. 1: Representation of a generation in load frequency control

As a single unit has been considered here, the participation factor $p f$ is taken to be 1 . As reference power $P_{\text {ref }}$ is obtained from security constraint power flow studies and not changed continuously, deviation in $P_{\text {ref }}$ i.e. $\Delta P_{\text {ref }}$ is to be taken taken as 0 . Hence, $u_{d k}$ becomes

$$
u_{d k}=-\int A C E(k)-\frac{\Delta f(k)}{R}
$$

The output of the governor dead-band at $k^{t h}$ instant, $y_{d k}$, depends on dead-band output at the previous time step $y_{d(k-1)}$ and present input $u_{d k}$. The governor dead-band output can be expressed as below.

$$
\begin{aligned}
& \text { If } u_{d k} \leq x_{d 0}+\frac{d}{2} \text { and } u_{d k} \geq x_{d 0}-\frac{d}{2} \\
& y_{d k}=y_{d(k-1)} \\
& \text { elseif } u_{d k} \geq x_{d 0}+\frac{d}{2} \\
& y_{d k}=u_{d k}-\frac{d}{2} \\
& \text { else if } u_{d k} \leq x_{d 0}-\frac{d}{2} \\
& y_{d k}=u+\frac{d}{2}
\end{aligned}
$$

The governor response is generally considered to be linear, and may be represented with a single time constant corresponding to the servo motor used to move the valves.

$$
\Delta \dot{P}_{v}=\frac{y_{d k}-\Delta P_{v}}{T_{g}}
$$

In compound turbines, the governor controls the steam flow to high pressure turbines. The steam chest, the inlet piping to the first turbine cylinder, the reheaters and the downstream crossover piping all introduce delays between the valve movement and change in steam flow to turbines. In this work, the total power is distributed between the high pressure (HP) and intermediate pressure (IP) turbines by fractions $F_{h p}$ and $\left(1-F_{h p}\right)$ respectively. The HP and IP turbines are represented with time constants $T_{t}$ and $T_{r}$ as

$$
\begin{gathered}
\Delta \dot{P_{t}}=\frac{\Delta P_{v}-\Delta P_{t}}{T_{t}} \\
\Delta \dot{P}_{r}=\frac{\left(1-F_{h p}\right) \Delta P_{t}-\Delta P_{r}}{T_{r}}
\end{gathered}
$$

\section{Output}

Though valve positions are usually monitored in plants, these measurements are generally not available outside the plant. The deviation in mechanical power $\Delta P_{m}$ may still be considered as an output in the estimation process, even though it is calculated from the electrical power and speed measurements. It may be noted that speed measurements may be noisy and hence the effect of the measurement noise has to be studied (results provided in Sec. VII).

$$
P_{m}=2 H\left(\frac{d \omega}{d t}\right)+P_{e}+D\left(\omega-\omega_{0}\right)
$$

Replacing $\omega$ with $\omega_{0}+\Delta \omega$

$$
P_{m}=2 H\left(\frac{d(\Delta \omega)}{d t}\right)+P_{e}+D(\Delta \omega)
$$

In UKF output, $\Delta P_{m}$ can be expressed in terms of the HP and IP turbine powers as follows

$$
\Delta P_{m}=F_{h p} \Delta P_{t}+\Delta P_{r}
$$

where $H$ is the generator inertia, $\omega$ is the unit speed, $\Delta \omega$ is the speed deviation from nominal, $P_{m}$ is the mechanical power, 
$D$ is the damping constant and $\omega_{0}$ and $P_{m 0}$ are the base speed and mechanical power. The states, inputs and measurements are

$$
\begin{gathered}
\text { states }=\boldsymbol{x}=\left[\begin{array}{lll}
\Delta P_{v} & \Delta P_{t} & \Delta P_{r}
\end{array}\right] \\
\text { inputs }=\mathbf{u}=\left[\begin{array}{cc}
A C E & \Delta f
\end{array}\right] \\
\text { output }=\mathbf{y}=\left[\Delta P_{m}\right]
\end{gathered}
$$

\section{Parameter Estimation Using Unscented KALMAN FILTER}

Unscented Kalman filter (UKF) [19] is a recursive filter in discrete-time domain which can model system nonlinearities as well as the measurement and process noise efficiently [19]. Consider a nonlinear system in discrete domain as

$$
\begin{gathered}
\boldsymbol{x}_{k}=\mathbf{f}\left(\boldsymbol{x}_{k-1}, \mathbf{u}_{k}\right)+\mathbf{q}_{k-1} \\
\mathbf{y}_{k}=\mathbf{h}\left(x_{k}\right)+\mathbf{r}_{k}
\end{gathered}
$$

where $\boldsymbol{x}$ is the state vector, $\mathbf{y}$ is the measurement vector, $\mathbf{q}$ and $\mathbf{r}$ are the state and measurement Gaussian noise with zero mean uncorrelated covariance matrices $\mathbf{Q}$ and $\mathbf{R}$, respectively. Functions $\mathbf{f}$ and $\mathbf{h}$ represents the system and measurement models in terms of system states and inputs $\mathbf{u}$. As droop and governor dead-band are to be estimated, these may be treated like states in this estimation process

$$
\text { states }=\boldsymbol{x}=\left[R d \Delta P_{v} \Delta P_{t} \Delta P_{r}\right]
$$

Step I: Initialization- The states and the covariance matrix is initialized at time instant $\mathrm{k}=0$, as

$$
\begin{gathered}
\boldsymbol{x}_{0}=E\left(\boldsymbol{x}_{0}\right) \\
\mathbf{P}_{0}=E\left(\left(\boldsymbol{x}_{0}-\hat{\boldsymbol{x}_{0}}\right)\left(\boldsymbol{x}_{0}-\hat{\boldsymbol{x}_{0}}\right)^{T}\right)
\end{gathered}
$$

where E denotes the expected value and " denotes estimated value.

Step II: Sigma Points Generation- For the $(n \times 1)$ state vector $\boldsymbol{x}_{k-1}$, and the corresponding covariance matrix $\mathbf{P}_{k-1}$, a set of $(2 n+1)$ vectors is formed, which are called sigma points, to capture the mean and covariance of $\boldsymbol{x}_{k-1}$.

$$
\begin{gathered}
\chi_{k-1}^{0}=\boldsymbol{x}_{k-1} \\
\chi_{k-1}^{i}=\boldsymbol{x}_{k-1}+\left(\sqrt{(n+\lambda) \mathbf{P}_{k-1}}\right)_{i} i=1 . . n \\
\chi_{k-1}^{n+i}=\boldsymbol{x}_{k-1}-\left(\sqrt{(n+\lambda) \mathbf{P}_{k-1}}\right)_{i} i=1 . . n
\end{gathered}
$$

where $\left(\sqrt{(n+\lambda) \mathbf{P}_{k-1}}\right)_{i}$ is the $i^{t h}$ column of the matrix $\left(\sqrt{(n+\lambda) \mathbf{P}_{k-1}}\right)$ and $\lambda=\alpha^{2}(n+\varrho)-n . \varrho$ is chosen as either $(3-n)$ or 0 and $\alpha$ is chosen between 0.001 and 1. All the sigma points may be written in a single $(n \times(2 n+1))$ matrix, as

$$
\chi_{k-1}=\left[\boldsymbol{x}_{k-1} . . \boldsymbol{x}_{k-1}\right]+\sqrt{(n+\lambda)}\left[0 \sqrt{\mathbf{P}_{k-1}}-\sqrt{\mathbf{P}_{k-1}}\right]
$$

Step III: Kalman filter state prediction- a) The sigma points are evaluated through the nonlinear prediction function in order to get the propagated sigma points.

$$
\hat{\chi}_{k}^{i}=f\left(\chi_{k-1}^{i}, \mathbf{u}_{k}\right)
$$

where $\chi_{k-1}^{i}$ is the $i^{t} h$ column of the matrix $\chi_{k-1}^{i}$ and the resulting $\hat{\chi}_{k}$ is an $n \times(2 n+1)$ matrix. The measurements being in discrete form, the nonlinear prediction function $\mathbf{f}$ is also evaluated in discrete form.

With all measurements in discrete form, the states are predicted at $k^{t h}$ time step from the estimated states of previous time step using the discrete form of the state equations given in Sec II. The time derivative of the parameters $R$ and $d$ are taken as 0 .

$$
\begin{aligned}
& x_{1}(k)=x_{1}(k-1) \\
& x_{2}(k)=x_{2}(k-1)
\end{aligned}
$$

The input to the governor dead-band is

$$
u_{d k}=\int A C E(k)-\frac{\Delta f(k)}{x_{1}(k-1)}
$$

The output of the governor dead-band $y_{d k}$ may be calculated from the present input $u_{d k}$ and the past output $y_{d(k-1)}$, as

$$
\begin{aligned}
& \text { If } u_{d k} \leq x_{d 0}+\frac{x_{2}(k-1)}{2} \text { and } u_{d k} \geq x_{d 0}-\frac{x_{2}(k-1)}{2} \\
& y_{d k}=y_{d(k-1)} \\
& \text { elseif } u_{d k} \geq x_{d 0}+\frac{x_{2}(k-1)}{2} \\
& y_{d k}=u_{d k}-\frac{x_{2}(k-1)}{2} \\
& \text { else if } u_{d k} \leq x_{d 0}-\frac{x_{2}(k-1)}{2} \\
& y_{d k}=u+\frac{x_{2}(k-1)}{2}
\end{aligned}
$$

Governor output $\Delta P_{v}\left(x_{3}\right)$ can be calculated from output of governor deadband $y_{d k}$

$$
x_{3}(k)=\left[y_{d k}-x_{3}(k-1)\right] \frac{\Delta t}{T_{g}}+x_{3}(k-1)
$$

$\Delta P_{t}\left(x_{4}\right)$ and $\Delta P_{r}\left(x_{5}\right)$ can be calculated as follows:

$$
\begin{gathered}
x_{4}(k)=\left[x_{3}(k-1)-x_{4}(k-1)\right] \frac{\Delta t}{T_{t}}+x_{4}(k-1) \\
x_{5}(k)=\left[\left(1-F_{h p}\right) x_{4}(k-1)-x_{5}(k-1)\right] \frac{\Delta t}{T_{r}}+x_{5}(k-1)
\end{gathered}
$$

b) The predicted state mean vector $\boldsymbol{x}_{k}^{-}$and the covariance matrix $\mathbf{P}_{k}^{-}$are computed from the propagated sigma points as

$$
\boldsymbol{x}_{k}^{-}=\sum_{i=0}^{2 n} \mathbf{W}_{i}^{m} \hat{\chi}_{k}^{i}
$$

$$
\mathbf{P}_{k}^{-}=\sum_{i=0}^{2 n} \mathbf{W}_{i}^{c}\left[\left(\hat{\chi}_{k}^{i}-\boldsymbol{x}_{k}^{-}\right)\left(\hat{\chi}_{k}^{i}-\boldsymbol{x}_{k}^{-}\right)^{T}\right]+\mathbf{Q}_{k-1}
$$

The weight in the above equation are calculated as follows: $W_{0}^{m}=\frac{\lambda}{n+\lambda} ; W_{i}^{m}=\frac{\lambda}{2(n+\lambda)}$

$W_{0}^{c}=\frac{\lambda}{n+\lambda}+\left(1-\alpha^{2}+\beta_{1}\right) ; W_{i}^{c}=\frac{\lambda}{2(n+\lambda)}$

For Gaussian distribution, $\beta_{1}$ is set to 2 for optimal performance.

Step IV: Kalman filter state correction- a) The predicted 
state mean vector and the covariance matrix calculated in the previous step, are used to update the sigma points (similar to Step II)

$$
\chi_{k}^{-}=\left[\boldsymbol{x}_{k}^{-} . . \boldsymbol{x}_{k}^{-}\right]+\sqrt{(n+\lambda)}\left[0 \sqrt{\mathbf{P}_{k-1}^{-}}-\sqrt{\mathbf{P}_{k-1}^{-}}\right]
$$

b) Measurements are predicted using the current sigma points and the covariance matrix

$$
\mathbf{Y}_{k}^{i-}=h\left(\chi_{k}^{i-}, u_{k}\right)
$$

c) The mean of the predicted measurements is calculated as

$$
\boldsymbol{\mu}_{k}=\sum_{i=0}^{2 n} \mathbf{W}_{i}^{m} \mathbf{Y}_{k}^{i-}
$$

d) Similar to the state covariance matrix in Step III, the measurement covariance matrix and the cross covariance of the state and measurement are estimated as

$$
\begin{gathered}
\mathbf{S}_{k}=\frac{1}{2 n} \sum_{i=0}^{2 n} \mathbf{W}_{i}^{c}\left[\left(\mathbf{Y}_{k}^{i-}-\boldsymbol{\mu}_{k}\right)\left(\mathbf{Y}_{k}^{i-}-\boldsymbol{\mu}_{k}\right)^{T}\right]+\mathbf{R}_{k} \\
\mathbf{C}_{k}=\sum_{i=0}^{2 n} \mathbf{W}_{i}^{c}\left[\left(\chi_{k}^{i-}-\boldsymbol{x}_{k}\right)\left(\mathbf{Y}_{k}^{i-}-\boldsymbol{\mu}_{k}\right)^{T}\right]
\end{gathered}
$$

e) The filter gain $\mathbf{K}_{k}$, the state $\boldsymbol{x}_{k}$, and the covariance $\mathbf{P}_{k}$ are computed from $\mathbf{y}_{k}$, the set of measurements at time instant $\mathrm{k}$.

$$
\begin{gathered}
\mathbf{K}_{k}=\mathbf{C}_{k} \mathbf{S}_{k}^{-1} \\
\boldsymbol{x}_{k}=\boldsymbol{x}_{k}^{-}+\mathbf{K}_{k}\left[\mathbf{y}_{k}-\boldsymbol{\mu}_{k}\right] \\
\mathbf{P}_{k}=\mathbf{P}_{k}^{-}-\mathbf{K}_{k} \mathbf{S}_{k} \mathbf{K}_{k}^{T}
\end{gathered}
$$

Step V: Memorizing backlash output- The governor dead-band, represented by a back-lash function, requires the knowledge of the previous output of the backlash. Therefore, after each step, using the estimated values of the states and parameters, the backlash output has to be calculated and stored, to be used in the UKF in the next time step.

$$
\begin{aligned}
& u_{d k}=-\int A C E(k)-\frac{\Delta f(k)}{x_{1}(k)} \\
& \text { If } u_{d k} \leq x_{d 0}+\frac{x_{2}(k)}{2} \text { and } u_{d k} \geq x_{d 0}-\frac{x_{2}(k)}{2} \\
& y_{d k}=y_{d(k-1)} \\
& \text { else if } u_{d k} \geq x_{d 0}+\frac{x_{2}(k)}{2} \\
& y_{d k}=u_{d k}-\frac{x_{2}(k)}{2} \\
& x_{d 0}=u_{d k}-\frac{x_{2}(k)}{2} \\
& \text { else if } u_{d k} \leq x_{d 0}-\frac{x_{2}(k)}{2} \\
& y_{d k}=u_{d k}+\frac{x_{2}(k)}{2} \\
& x_{d 0}=u_{d k}+\frac{x_{2}(k)}{2} \\
& \text { end }
\end{aligned}
$$

The difference between (15) and (32) is to be noted that in (32) the midposition of the governor dead-band is also updated which will be used in (15) in next time step. $y_{d k}$ is the deadband output at $k^{t h}$ instant and is stored in variable $y_{d(k-1)}$ to use in the next step.

$$
y_{d(k-1)}=y_{d k}
$$

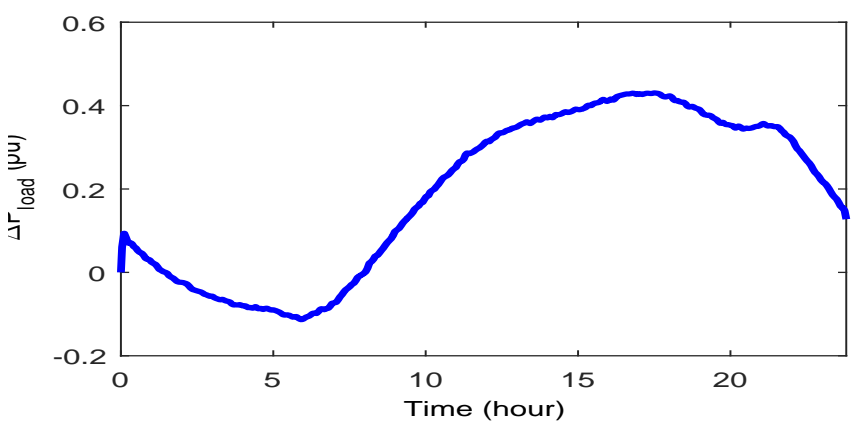

Fig. 2: Daily load deviation curve. Base load is taken as the load at the starting point of the day

UKF parameters: There are several parameters that need to be initialized before running the estimation algorithm- Process covariance matrix

$\mathbf{Q}=q^{2} \operatorname{diag}([0 ; 0 ; 1 ; 1 ; 1])$

State covariance matrix

$\mathbf{P}=\operatorname{diag}([p(1) ; p(2) ; p(3) ; p(4) ; p(5)])$

Measurement covariance: $\mathbf{R}$

Therefore, total seven parameters of UKF are to be initialized, as given by

$p p=[q, p(1), p(2), p(3), p(4), p(5), \mathbf{R}]$

\section{RESUlTS}

The proposed method was first tested on a linearized two area power system model (similar to Fig. 1), whose parameters are given in the appendix. As the estimation is supposed to be done with ambient measurements, rather than any particular disturbance, the load in area 1 of the test system was changed continuously. The load data was taken from the daily load curve of New York ISO [20], on $19^{\text {th }}$ June, 2016. As load power deviation $\Delta P_{\text {load }}$ is used in the control, it was obtained as the deviation from the initial load at the start of the day and then taken as per-unit (with initial load as the base), shown in Fig.2

\section{A. Estimation for legacy thermal generator with larger dead- band}

Many of the legacy thermal generator have dead-band in the range of $0.05 \%-0.06 \%$, and this has been implemented with backlash in MATLAB Simulink. The proposed method has been tested to estimate this dead-band width and droop. Actual values of the parameters were selected as droop $=2.4$ $H z / p u M W$ and dead-band $=0.015 p u M W$. The estimation program took almost $500 \mathrm{~s}$ ( $8 \mathrm{~min}$ ) of data to converge to their actual values from initial values of $1.5 \mathrm{~Hz} / \mathrm{puMW}$ and $0.01 \mathrm{puMW}$, as shown in Fig.3. After running the estimation program for one hour, the final error was $-1.3 \%$ for droop and $0.09 \%$ for dead-band width which is considerably small for all practical purposes. The parameters for UKF were set at $p p=\left[\begin{array}{llllll}10^{-6} & 10^{-3} & 10^{-7} & 1 & 1 & 10^{-6}\end{array}\right]$ 

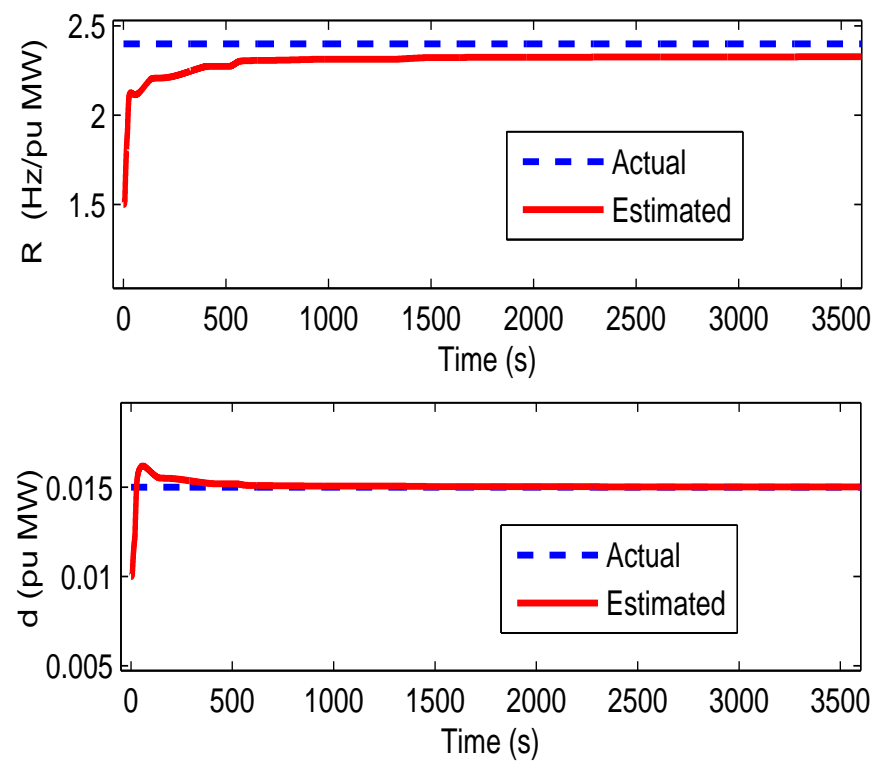

Fig. 3: Estimation of parameters for old thermal generators with larger deadband

\section{B. Performance on actual nonlinear model}

The proposed method was tested on Kundur's two area test system [12]. Description of the turbine governor is given below,

i)Turbine has been modelled as tandem compound, as this is large in number in the industry.

ii)Presently available professional simulations tools representing the governor turbine models in detail (like PSS/ETGOV5 model), have the options to include deadband in frequency measurement, but not the governor deadband. During simulation in this paper, intentional frequency dead-band was put to zero and unintentional dead-band is zero (as there is no option to include it). Therefore, the estimated governor deadband should converge to 0 in this case.

iii) the single mass model was considered, the dynamics under consideration is very slow as compared to torsional dynamics.

iv)boiler pressure was considered as constant, as the estimation was done with ambient data, not big disturbance.

As measurements were taken from the generator inputs and outputs, the performance of the method is independent of the rest of the system. A load of $1 \%$ of the generator rating (900 MVA) was switched on and off every 50s. The mechanical power was calculated as given in Sec-II. The calculated mechanical power matched the actual mechanical power well as shown in Fig.4 except the small error caused by electrical transients just after the load change.

Estimation of droop and dead-band width - With initial values for the droop and the dead-band width selected to be $10 \%$ and $0.005 p u M W$, the estimation algorithm was run for 500s. The droop estimate settled to $4.82 \%$ against an actual droop of $5 \%$ (error $-3.6 \%$ ). As there was no dead-band in the governor model, the estimated governor dead-band was estimated to be almost zero i.e. $1.23 * 10^{-6}$, as shown in Fig.5.

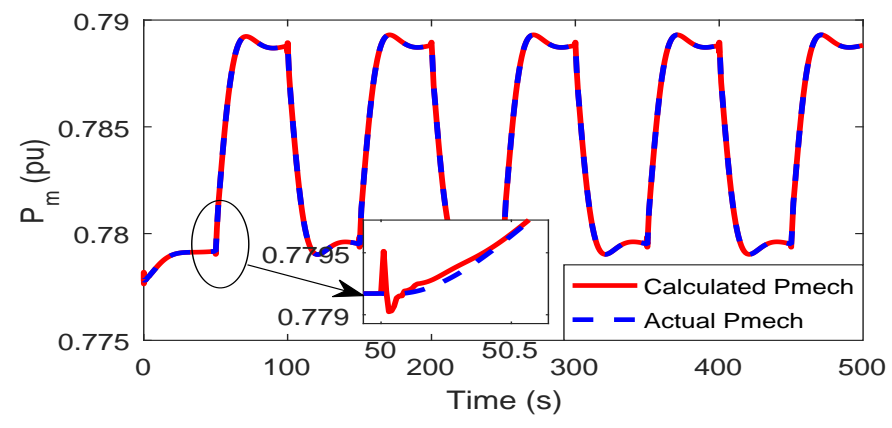

Fig. 4: Actual mechanical power and mechanical power calculated from electrical power and speed)
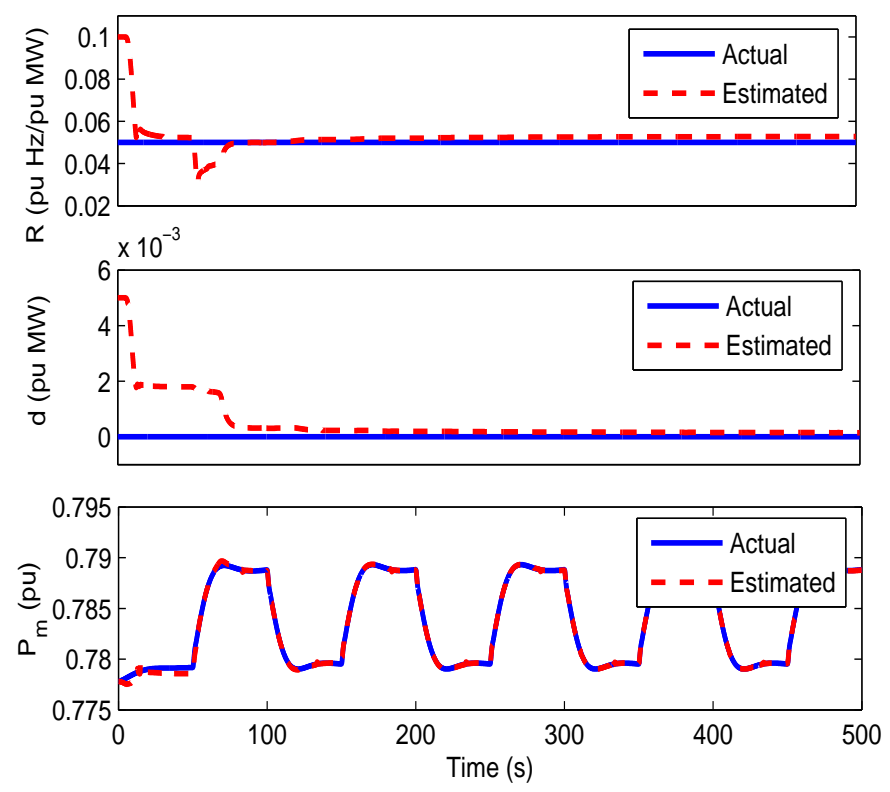

Fig. 5: Estimation of parameters for fully nonlinear thermal generator with zero deadband (taken from MATLAB Simulink)

The set parameters in the UKF were

$$
p p=\left[10^{-6}, 10^{-4}, 10^{-7}, 1,1,1\right]
$$

\section{COMparison With Recursive Least SQuare}

The performance of the proposed method was compared with the recursive least square technique (RLS) proposed in [7] and summarized in Table I.

It can be concluded from the comparison that UKF can be more accurate in presence of non-linearities although with higher data frequency requirements. The parameters were also estimated using nonlinear least square technique (fmincon in MATLAB) and the method was inaccurate with measurement noise. The results have not been included because of space constraints. 
TABLE I: Comparison between proposed method and RLS [7] $-[10]$

\begin{tabular}{|c|c|c|}
\hline Feature & UKF & RLS \\
\hline Objective & $\begin{array}{l}\text { Estimates droop and } \\
\text { governor dead-band } \\
\text { width of individual } \\
\text { generator }\end{array}$ & $\begin{array}{l}\text { Estimates area frequency char- } \\
\text { acteristic } \beta \text {, which can be ap- } \\
\text { proximated as droop. Gener- } \\
\text { ally droop is close to } 95 \% \text { of } \\
\beta\end{array}$ \\
\hline $\begin{array}{l}\text { Data } \\
\text { used }\end{array}$ & $\begin{array}{l}\text { PMU data of } \\
\text { generator buses, } \\
\text { tie line power flow } \\
\text { and } \int A C E \text { signal } \\
\text { sent to generator as } \\
\text { per the participation } \\
\text { factor. }\end{array}$ & $\begin{array}{ll}\text { SCADA measurements } & \text { for } \\
P_{g}, P_{\text {tie }}, P_{\text {load }}, \Delta f \text { etc. } & \end{array}$ \\
\hline $\begin{array}{l}\text { Data } \\
\text { fre- } \\
\text { quency }\end{array}$ & $30-120 \mathrm{~Hz}$ & Once in 2 or 3 seconds \\
\hline $\begin{array}{l}\text { Estimation } \\
\text { Error }\end{array}$ & $\begin{array}{l}\text { Error in droop es- } \\
\text { timation was } 3.73 \% \\
\text { when deadband was } \\
\text { also considered un- } \\
\text { known. }\end{array}$ & $\begin{array}{l}\text { Error in estimated } \beta \text { (primary } \\
\text { frequency characteristic) was } \\
\text { around } \\
\text { a) } 2 \% \text { without considering } \\
\text { deadband and } \\
\text { b) } 41 \% \text { with deadband } \\
\text { included in simulation. }\end{array}$ \\
\hline $\begin{array}{l}\text { Possible } \\
\text { Difficul- } \\
\text { ties }\end{array}$ & $\begin{array}{l}\text { a) Mechanical power } \\
\text { estimation. As } \\
\text { mechanical power } \\
\text { is generally not } \\
\text { measured, it has to be } \\
\text { estimated/calculated } \\
\text { and that may be } \\
\text { erroneous. } \\
\text { b)availability of } \\
\text { reliable generator } \\
\text { PMU data. }\end{array}$ & $\begin{array}{l}\text { a) RLS can't handle nonlinear- } \\
\text { ities like deadband and GRC. } \\
\text { Therefore, accuracy drops to a } \\
\text { very low value with nonlinear- } \\
\text { ities. } \\
\text { b)SCADA data is very often } \\
\text { not accurate, and state estima- } \\
\text { tion is run less frequently (typ- } \\
\text { ically in } 5 \text { s interval). } \\
\text { c)Measured load power is not } \\
\text { the } P_{l o a d} \text { used in load fre- } \\
\text { quency control, rather it is } \\
P_{l o a d} \times(1-D \Delta f) \text {. The ac- } \\
\text { curate measurement of } P_{l o a d} \\
\text { is difficult to obtain. } \\
\text { d)In a large control area, the } \\
\text { local frequency is not same at } \\
\text { different buses. Considering a } \\
\text { single frequency for an entire } \\
\text { area may cause error in prac- } \\
\text { tice, especially when the gen- } \\
\text { erators in a control area are not } \\
\text { fully coherent. }\end{array}$ \\
\hline Utility & $\begin{array}{l}\text { a) Adaptive frequency } \\
\text { bias setting } \\
\text { b) Estimating droop } \\
\text { of each generator, can } \\
\text { be considered parallel } \\
\text { for calculating droop } \\
\text { of the entire area. }\end{array}$ & $\begin{array}{l}\text { a) Adaptive frequency bias set- } \\
\text { ting } \\
\text { b) Estimating primary fre- } \\
\text { quency reserve and droop } \\
\text { available. }\end{array}$ \\
\hline
\end{tabular}

\section{UTILITY}

\section{A. Adaptive Tuning of Frequency Bias Setting}

Frequency bias is generally set as per different performance indices for different frequency biases for step changes in load. Impact of bias setting on the performance of the LFC system for continuous load changes is more important, rather than rare events. A continuous load change was applied to area 1 as shown in Fig.2 and the following indices were calculated for 1 hour period and shown in Fig.6.

$$
\begin{gathered}
\text { RMS Control Effort }=\Delta \text { Effort }_{r m s}= \\
\sqrt{\frac{\int_{0}^{T}\left(u_{d}-P_{\text {load }}\right)^{2} d t}{T}}, \quad \text { RMS } \Delta \text { Ptie }=\Delta \text { Ptie }_{r m s}=
\end{gathered}
$$

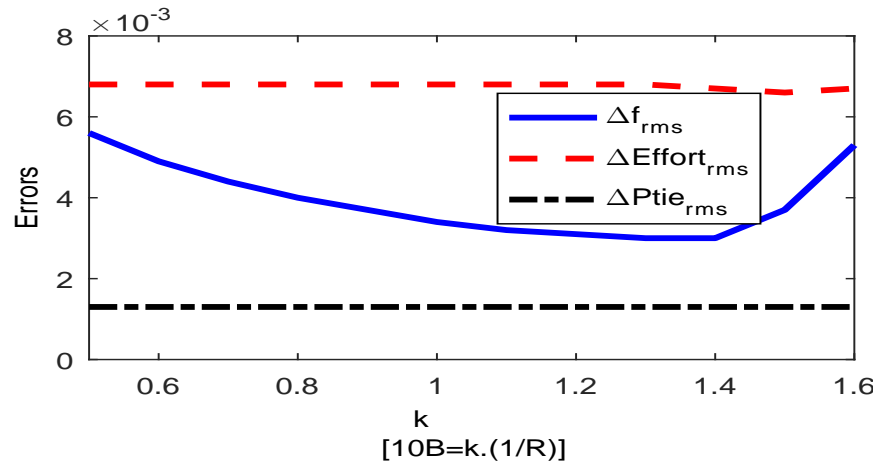

Fig. 6: Errors in control effort,tie line power deviation andfrequency error for different values of frequency bias constant (10B). 10B has been expressed as $\frac{1}{R}$ multiplied and $\mathrm{k}$ varies from 0.5 to 1.6

$\sqrt{\frac{\int_{0}^{T} \Delta P t i e^{2} d t}{T}}, R M S \Delta f=\Delta f_{r m s}=\sqrt{\frac{\int_{0}^{T} \Delta f^{2} d t}{T}}$

For an ideal load (of same area) tracking performance, the values all these indices should tend to zero. If there was no governor dead-band (as considered in [7]), then the frequency bias $10 B$ could be set equal to $\beta=\frac{1}{R}+D \approx \frac{1}{R}$.In presence of dead-band, the value of $\beta$ obtained from RLS was close to $60 \%$ of the actual $\beta$ [7]. It was found that frequency bias constant should be set to $\beta$ without governor dead-band [7] and lesser than $\beta$ with governor dead-band. It can be seen from Fig.6 that the tie line power and control effort remains almost the same for different values of frequency bias, but the frequency deviation is minimum when frequency bias $10 \mathrm{~B}$ is equal to $\left(1.3 \times \frac{1}{R}\right)$ which differs from the optimum frequency bias obtained for step change in load [7], [12]. As this setting is not universal and varies with governor dead-band width, droop and so on, these parameters should be accurately monitored.

\section{B. Estimating Primary Frequency Reserve Requirement}

Generally, all generators have intentional and unintentional dead-bands, but the dead-bands are usually not considered while studying frequency disturbances. However, both intentional and unintentional dead-band have significant impacts on the PFR of the system. For instance, the Eastern Interconnection has an average area frequency response characteristic $\beta$ of $2363 \mathrm{MW} / 0.1 \mathrm{~Hz}$. A typical dead-band of $0.06 \%$ i.e. $36 \mathrm{mHz}$ amounts to $851 \mathrm{MW}$. Therefore, in a pessimistic case, generation loss of up to $851 \mathrm{MW}$ will not cause any primary frequency response because of presence of deadband. In that case, the responsibility of correcting the system frequency would ultimately rest with the slow-acting secondary frequency controllers and thus frequency response would deteriorate [2].

\section{Impact of Measurement Noise on Estimation}

The mechanical power has been calculated from the electrical power and speed measurements (eq.(6)). However, the generator speed measurement can be noisy and inaccurate. Therefore, mechanical power was calculated using measured 
TABLE II: Noise Level Vs Estimation Error

\begin{tabular}{|c|c|}
\hline Noise Level in speed & Estimation Error(Actual $\mathrm{R}=2.4, \mathrm{~d}=0.015)$ \\
\hline $1 \%$ & Estimated: $\mathrm{R}=2.382,0.015$ \\
SNR $=40.01 \mathrm{db}$ & Error $\%=0.7576,-0.0692$ \\
\hline $5 \%$ & Estimated: $\mathrm{R}=2.386,0.015$ \\
SNR $=25.99 \mathrm{db}$ & Error $\%=0.5677,-0.0655$ \\
\hline $10 \%$ & Estimated: $\mathrm{R}=2.385,0.015$ \\
$\mathrm{SNR}=25.99 \mathrm{db}$ & Error $\%=0.6169,-0.0827$ \\
\hline
\end{tabular}

speed deviation added with $10 \%$ random noise (Fig.7b). The noise level in the speed signal was varied, and the error in the estimated parameters were found to be below 1\% (TableII).

\section{IMPACT OF GRC ON ESTIMATION}

The presence of generation rate constraint (GRC) may affect the estimation accuracy. The rate of change of generator power is limited because of turbine and boiler response rate constraints. For thermal generators, it may be taken as $10 \%$ per minute (i.e. $0.0017 \mathrm{pu} / \mathrm{s}$ ). GRC has been modeled as an open loop model [21], as shown in Fig.8.

The mechanical power without considering the GRC, may be calculated as:

$$
\Delta \dot{P}_{m}^{\prime}=F_{h p} \Delta P_{t}+\Delta P_{r}
$$

Suppose the maximum rate at which generator power can increase or decrease are $+r$ and $-r$. Mathematically, the GRC is represented as:

$$
\begin{aligned}
& \text { If } \Delta \dot{P}_{m}^{\prime}>+r \\
& \Delta \dot{P}_{m}^{\prime}=+r \\
& \text { else if } \Delta \dot{P}_{m}^{\prime}<-r \\
& \Delta \dot{P}_{m}^{\prime}=-r \\
& \text { else } \\
& \Delta \dot{P}_{m}^{\prime}=\Delta \dot{P}_{m}^{\prime}
\end{aligned}
$$

If $\Delta P_{m(k)}$ and $\Delta P_{m(k-1)}$ are the mechanical power at $k^{t h}$ instant and $(k-1)^{\text {th }}$ instant

$$
\Delta P_{m(k)}=\Delta P_{m(k-1)}+\Delta \dot{P}_{m}^{\prime} \Delta t
$$

The dynamic equations for the rest of the system will remain same as explained in Sec. III.

Changes in UKF Implementation: As GRC is a design parameter, it would be known to the system operator and cannot be changed during daily operation. Accordingly, the GRC was included as a known parameter during estimation. As GRC has been modeled after the turbine-governor blocks, the state equations corresponding to the turbine-governor remain unchanged. However, it affects the net mechanical power, which has been considered as an output in the UKF. Therefore, the output equations in Sec II (eqn.(7)) and Sec III (eqn. 24) will change and the mechanical power has to be modified as in (36). Just like governor deadband, the GRC also depends on the past output (mechanical power at previous instant), and therefore just like step $\mathrm{V}$, Sec. III, the $\Delta P_{m(k)}$ has to be stored for using as $\Delta P_{m(k-1)}$ in the next time step.

The efficiency of the estimation algorithm in the presence of GRC was tested, and the results are shown in Fig.9. At time $500 \mathrm{~s}, 0.5 \%$ load was switched on which resulted in a rapid rise

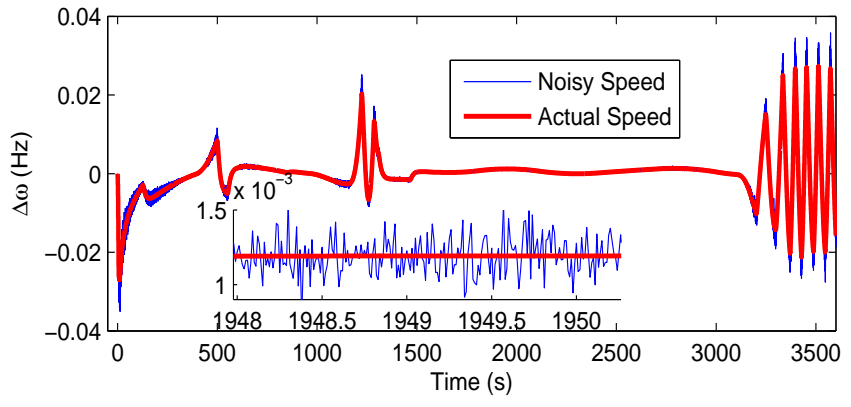

(a) Actual speed deviation and noisy speed signal

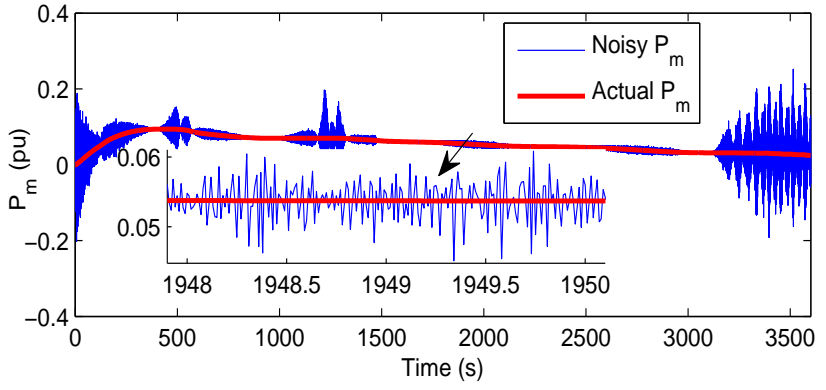

(b) Actual and calculated mechanical power

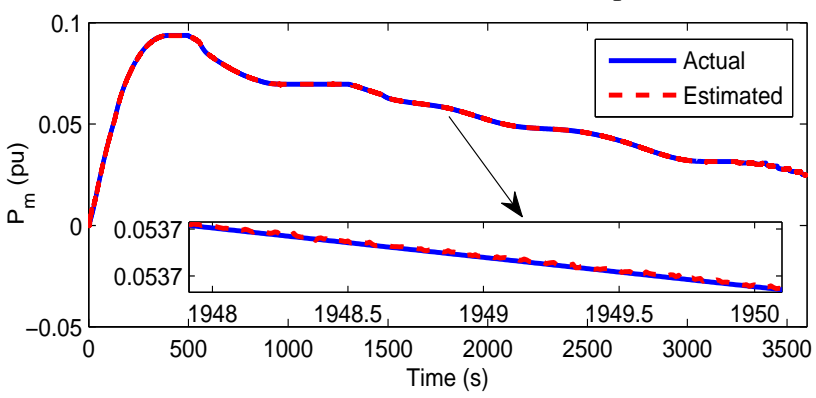

(c) Actual and estimated $\Delta P_{m}$

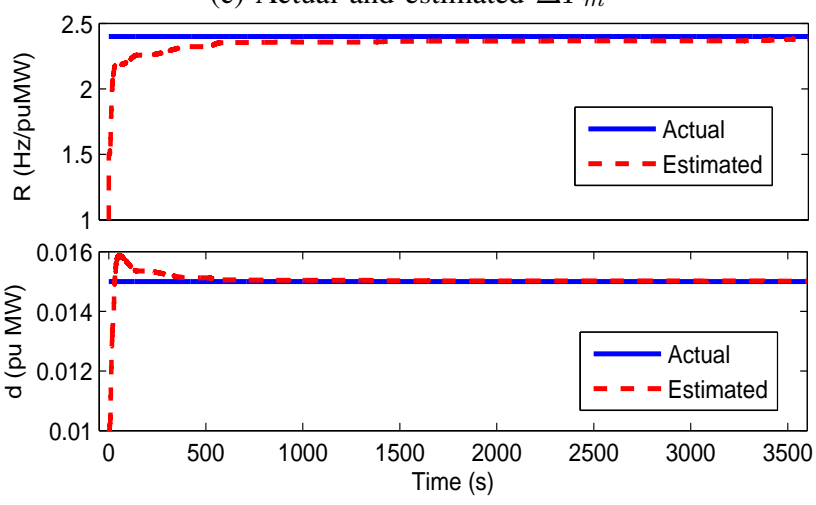

(d) Droop and deadband estimated with UKF

Fig. 7: Impact of measurement noise on estimation performance

in $\int A C E$ sent to the generator from the control centre. The rate of rise of mechanical power before GRC block reached $0.003 \mathrm{pu} / \mathrm{s}$ (i.e. $18 \%$ per minute) while the GRC limit was $0.0017 \mathrm{pu} / \mathrm{s}$. Hence, the GRC came into action and limited the rate of rise of generator power to $0.0017 \mathrm{pu} / \mathrm{s}$, as shown in Fig.9(a).

It may be observed from Fig. 9 that during the GRC operation following a transient, there is negligible error between the 


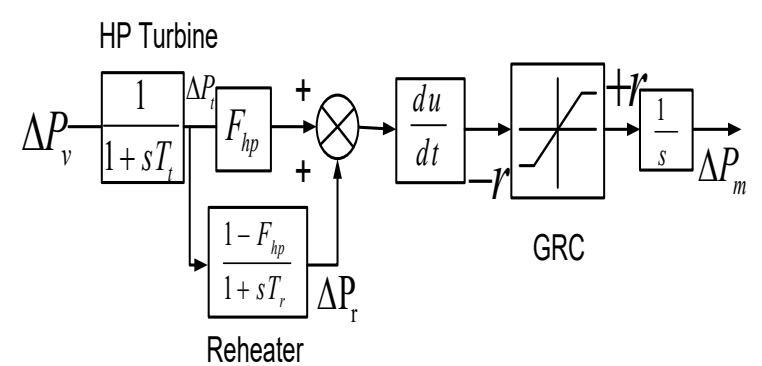

Fig. 8: Inclusion of GRC in turbine-governor model

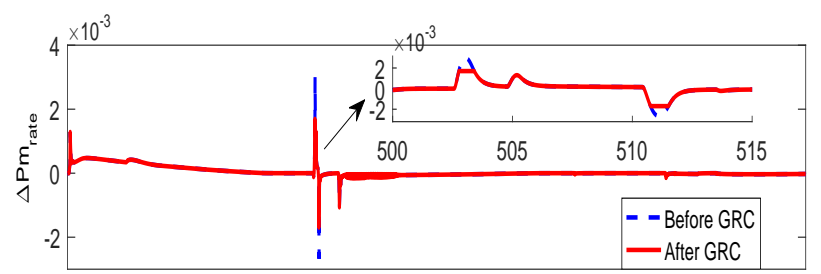

(a)
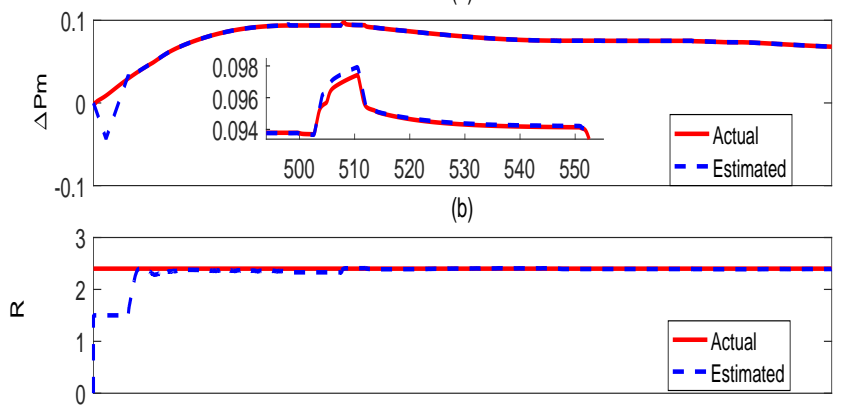

(c)

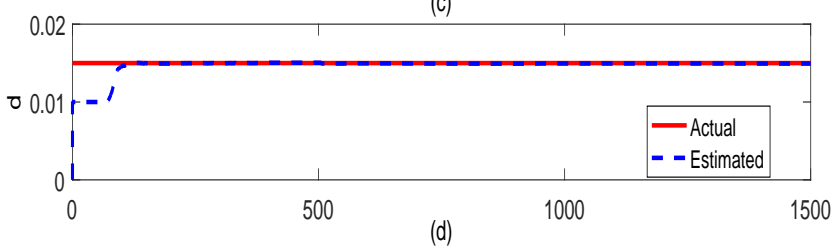

Fig. 9: Impact of GRC on estimation

estimated and actual mechanical power and the method is able to estimate the droop and governor deadband in the presence of GRC.

\section{BAD DATA DETECTION}

There may be bad data in measurements like frequency $\Delta f$, generator speed $\Delta \omega$, electrical power $P_{e}$ or in updating of generation raise/lower signal $\int A C E$. As $\Delta P_{m}$ is calculated from $\Delta \omega$ and $\Delta P_{e}$, erroneous $\Delta \omega$ and $\Delta P_{e}$ may affect the mechanical power $\Delta P_{m}$ calculation. As $\Delta P_{m}$ is the only measurement in this estimation process using UKF and no redundant measurement available, any bad data in it may cause failure of the entire estimation process. Therefore, any bad data in inputs or measurement has to be detected and estimation may be paused if needed.

\section{A. Preprocessing of data in measurements}

Generally the inputs in Kalman filter are preprocessed (filtered) before being used for estimation. Therefore, bad data and noise are most likely to be filtered out. However the method should be robust to any outlier present in the measurements, especially the inputs.

1) Preprocessing Bad Data in frequency measurement: Maximum possible sample to sample difference in frequency measurement is observed after a large disturbance like generator outage or tie line tripping. Considering $1 \%$ generation change (which can be because of the biggest generator or tie line tripping), maximum sample to sample frequency difference was $0.0006 \mathrm{~Hz}$ for the test system given in Appendix (100 samples/s).

$$
\left|\Delta f_{k}-\Delta f_{k-1}\right| \leq 0.0006 H z
$$

Considering maximum $10 \mathrm{mHz}$ error in frequency measurement [22]- [23], in the worst case sample to sample frequency difference becomes

$$
\left|\Delta f_{k}-\Delta f_{k-1}\right| \leq 0.0006+2 * 0.01 \mathrm{~Hz}=0.0206 \mathrm{~Hz}
$$

If frequency measurement at $k^{t h}$ instant deviates from the previous measurement by more than $0.0206 \mathrm{~Hz}$, frequency measurement at $k^{t h}$ instant is discarded and replaced with linearly extrapolated value from the previous measurements.

As frequency does not change instantaneously, most bad data can be easily filtered out.

2) Preprocessing of Bad Data in ACE measurement: Maximum error in $\int A C E$ can be taken as the $0.05 p u$, as power reference in generators is changed in small steps of $5-10 M W$ because of GRC and other constraints. $10 M W$ step would be approx. $0.05 \mathrm{pu}$ for a $210 \mathrm{MW}$ generator and smaller for bigger units. If there is any sample to sample difference in $\int A C E$ higher than $0.05 p u$, then previous data may be used.

\section{B. Bad Data Detection Tests}

Bad data in inputs or output of UKF impacts the performance differently. There are three different tests to detect bad data [24], as given below:

1) Test 1: Innovation magnitude test: - Considering $2 \sigma$ bound, $95 \%$ of the innovation samples $\left(y_{k}-\mu_{k}\right)$ should lie within $\pm 2 \sqrt{S_{k}}$ (see eqn. (26)). Innovation and normalized innovation may be defined as:

Innovation at $k^{t h}$ instant: $\nu_{k}=\left(y_{k}-\mu_{k}\right)$

Normalized innovation at $k^{\text {th }}$ instant: $\bar{\nu}_{k}=\frac{\left(y_{k}-\mu_{k}\right)}{\sqrt{S_{k}}}$

Therefore, $95 \%$ of the $\bar{\nu}_{k}$ should lie within \pm 2 .

2) Test 2: Normalized Innovation $\chi^{2}$ Squared test: Innovation sequence should have zero mean. To check the unbiasedness, compute normalized innovation squared

$$
\overline{\nu_{k}^{2}}=\nu_{k}^{T} S_{k}^{-1} \nu_{k}
$$

This $\overline{\nu_{k}^{2}}$ should be $\chi^{2}$ in $m$ degree of freedom, where $m=$ degree of freedom. In this case, there is only one independent measurement, hence $m=1$. Thus, $E\left(\overline{\nu_{k}^{2}}\right)=m=1$ and the mean of $\overline{\nu_{k}^{2}}$ is $\bar{\nu}_{a v}=\frac{1}{N} \sum_{k=1}^{N}{\overline{\nu_{k}}}^{2}$ 

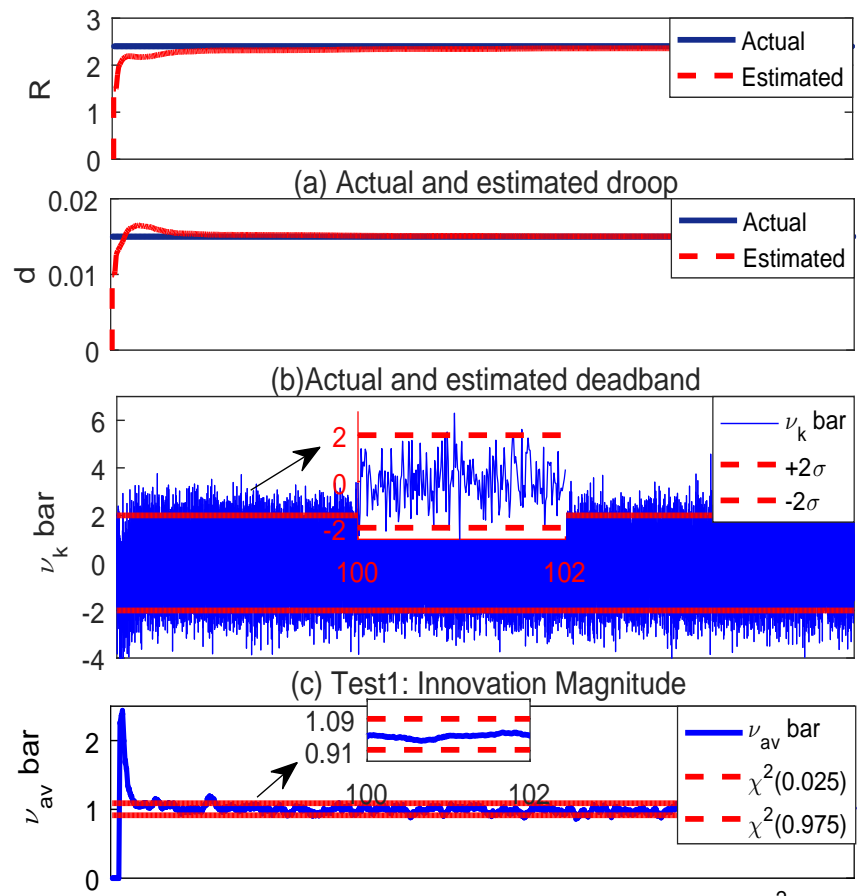

(d) Test 2: Average normalized Innovation Squared $x^{2}$ test

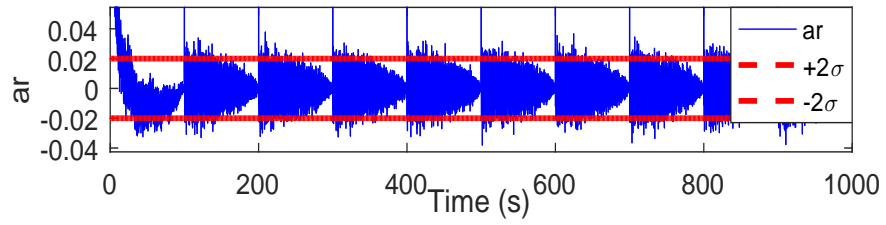

(e) Test 3: Autocorrelation of innovation

Fig. 10: Bad data tests on correct (without bad data) measurement

To check unbiasedness, for data length $\mathrm{N}$, we need to verify that $N \bar{\nu}_{a v}$ lies within $\left[r_{1}, r_{2}\right]$ such that probability

$$
P\left(N \bar{\nu}_{a v}\right) \in\left[r_{1}, r_{2}\right]=1-\alpha
$$

If $\alpha=0.05$ and $N=1000, N \bar{\nu}_{a v}$ is less than the critical value $\chi_{1000,0.05}^{2}$ with probability $(1-\alpha)$ or $95 \%$ confidence. $\chi_{1000,0.05}^{2}$ can be found out from statistical table for $\chi^{2}$ distribution.

3) Test 3: Innovation whiteness test: Innovation $\nu$ should be white noise unless there is any fault in the outputs or inputs. Whiteness of the $\nu$ may be tested from autocorrelation test of $\nu$ :

$$
\operatorname{ar}(\tau)=\frac{1}{\tau} \sum_{i=0}^{N-\tau-1} \nu_{k} \nu_{k+\tau}
$$

$\operatorname{ar}(\tau)$ has highest value at $\tau=0$, hence ar is normalized with $\operatorname{ar}(0) \cdot \operatorname{ar}(\tau)=\frac{\operatorname{ar}(\tau)}{\operatorname{ar}(0)}$ should be randomly distributed with mean 0 , variance $\frac{1}{N}$. Considering $\pm 2 \sigma$ bound, $95 \%$ of the values of ar should lie within $\pm \frac{2}{\sqrt{N}}$ bound.

\section{Results without any bad data}

First, all the three tests are carried for the operating condition and test system used in Sec-V, measurements without any bad data and the results are given below in Fig.10.

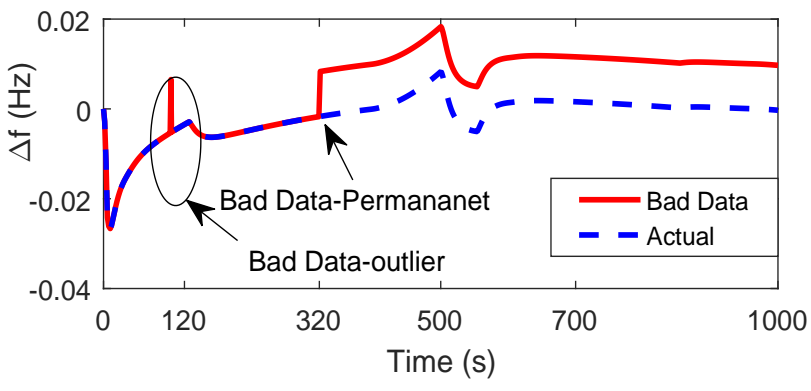

Fig. 11: Actual $\Delta f$ and $\Delta f$ corrupted with bad data. $10 \mathrm{mHz}$ outlier and steady state bad data added to actual frequency at 120 s and 320 s

It can be seen from the Fig. 10 -(a) and (b) that the estimated parameter values almost converged to the actual values. From Fig. 10-c it can be seen that most of the normalized innovation samples $\left(\bar{\nu}_{k}\right)$ lie within \pm 2 , as discussed in Sec IX(B). If a moving window of 1000 data samples (at 100 samples per s from PMU) is considered to check the chi - square distribution with $95 \%$ confidence, the upper and lower bound are obtained from the chi-square table $\chi_{1000,0.025}^{2}=1089.531$ and $\chi_{1000,0.975}^{2}=914.257$. It means that $1000\left(\bar{\nu}_{a v}\right)$ will lie within this band with $95 \%$ probability or $\left(\bar{\nu}_{a v}\right)$ will lie within 1.089 and 0.914 . From test 2 in Fig.10(d) it may be seen that average normalized innovation squared (i.e. $\left(\bar{\nu}_{a v}\right)$ ) follows the chi squared distribution and lies within a certain upper and lower band- 1.0895 and 0.9143 . Normalized autocorrelation of the innovation was calculated for each $100 \mathrm{~s}$ window. From Fig.10-e, it can be seen that the autocorrelation of the innovation ar lies within $\pm 2 \sigma=$ $\pm \frac{2}{\sqrt{N}}= \pm \frac{2}{\sqrt{10000}}= \pm 0.02$ (total number of samples $=10000$, at $100 \mathrm{samples} / \mathrm{s}$ for $100 \mathrm{~s}$ window).

\section{Estimation with Bad Data}

Estimation was done in the presence of bad data in input $\triangle f$ and $\int A C E$ and output $\Delta P_{m}$ for separate outliers and continuous measurement errors.

1) Bad Data in $\Delta f$ : There may be two types of bad data - temporary outlier such as single erroneous data in a large data window and continuous errors in measurement. Bad data was included in $\Delta f$ as shown in Fig.11

The proposed algorithm was run to estimate the parameters in presence of the bad data and results are provided in Fig.12. It can be seen from Fig.12 that estimation of $R$ and $d$ was almost accurate until $500 \mathrm{~s}$ and becomes poor thereafter $\left(\right.$ Fig.12(a)\&(b)), the normalized innovation magnitude $\bar{\nu}_{k}$ and average normalized innovation squared $\bar{\nu}_{a v}$ are within their upper and lower bounds until $325 \mathrm{~s}$ and goes beyond this thereafter $($ Fig.12 $(c) \&(d))$ and the innovation is white noise only for the first three window $(0-100,101-200,201-300)$ as the autocorrelation of the innovation lies within the upper and lower bounds (Fig.12-e).

It may be concluded that the estimation method worked perfectly for the single bad data at 120 s but was inaccurate during permanent measurement error from $325 \mathrm{~s}$ till end. A 


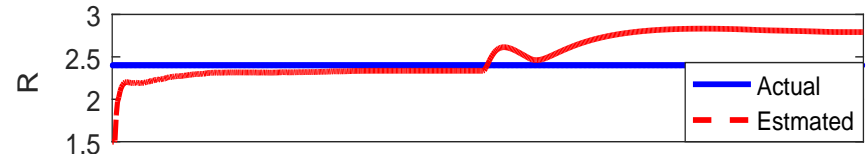

(a) Actual and estimated droop

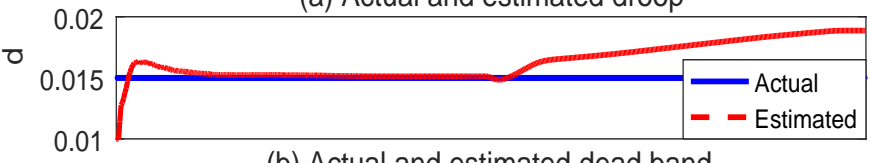

(b) Actual and estimated dead band

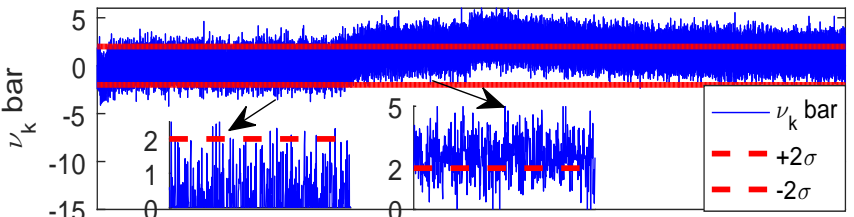

(c) Innovation magnitude test

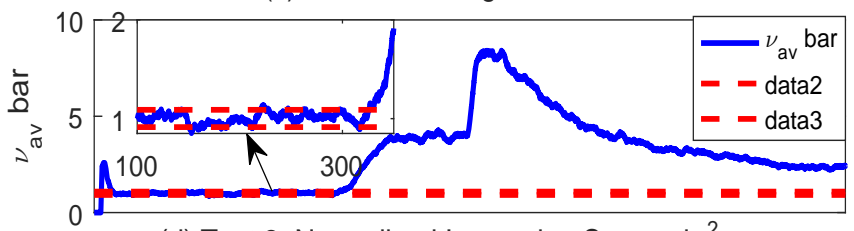

(d) Test 2: Normalized Innovation Squared $\chi^{2}$ test

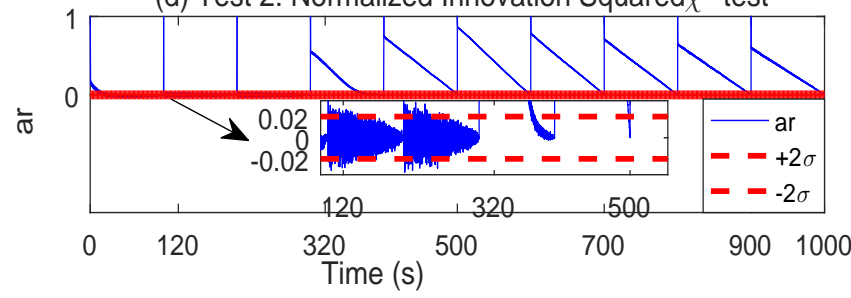

(e) Test 3: Autocorrelation Test

Fig. 12: Impact of bad data in input $\Delta f$ on UKF performance and detection of bad data

single bad data or outlier in $\Delta f$ measurement at 120 s did not cause any noticeable change in the three performance tests as well as estimation accuracy, hence the method is robust to it.

Similar conclusion can be drawn for bad data in input $\int A C E$, results not provided because of space constraints.

2) Bad Data in Pm: Bad data in $\Delta P_{m}$ may arise from erroneous $\Delta \omega$ measurements or $\Delta P_{e}$ during transients, communication channels etc. A single outlier bad data and permanent bad data was added to the $\Delta P_{m}$ measurement at $120 \mathrm{~s}$ and $320 s$ respectively before using in UKF, as shown in Fig.13.

The proposed algorithm was run to estimate the parameters in presence of the bad data in $\Delta P_{m}$. Detection of Bad data was also done as shown in Fig.14.

It can be seen from that estimation of $R$ and $d$ was almost accurate until 320s and becomes poor thereafter (Fig.14$\mathrm{a} \& \mathrm{~b})$, the normalized innovation magnitude $\left(\bar{\nu}_{k}\right)$ and average normalized innovation squared $\bar{\nu}_{a v}$ are within their bounds until 320s $(F i g .14(c) \&(d))$ and the innovation is white noise for only the first three window (0-100, 101-200, 201-300).

Unlike bad data in input $\Delta f$, even a single outlier bad data in $\Delta P_{m}$ can be detected from the innovation magnitude test, as shown in Fig.14(c) at $\mathrm{t}=120 \mathrm{~s}$. As per $2 \sigma$ confidence level, $95 \%$ of normalized innovation samples should lie within \pm 2 . Therefore a threshold of slightly higher value than +2 or slightly lower than -2 can be chosen. A threshold bound of

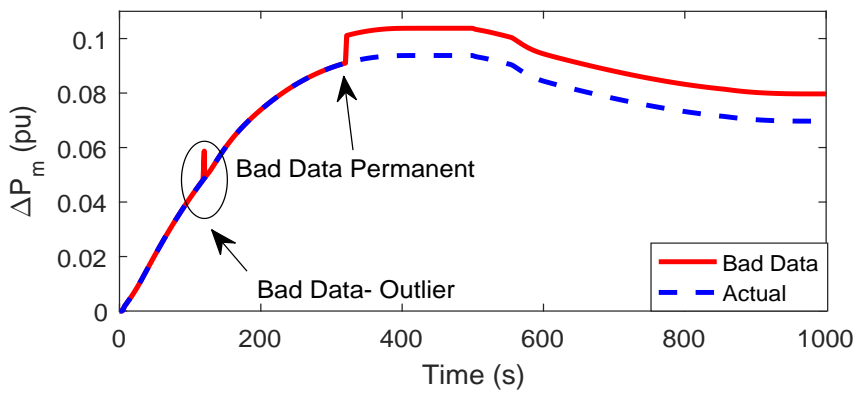

Fig. 13: Actual $\Delta P_{m}$ and $\Delta P_{m}$ measurement corrupted with bad data. $0.01 p u$ outlier and steady state bad data added to actual $\Delta P_{m}$ at $120 \mathrm{~s}$ and $320 \mathrm{~s}$

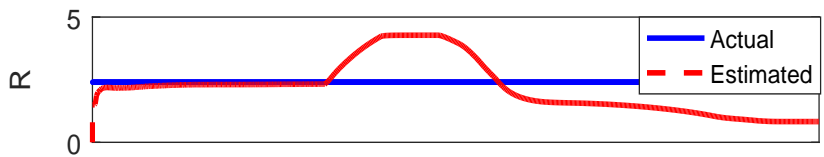

(a) Actual and estimated droop

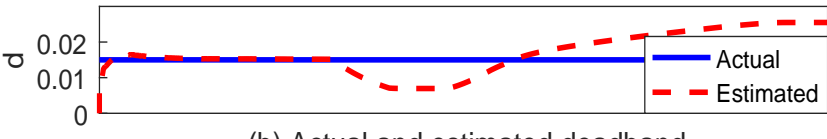

(b) Actual and estimated deadband

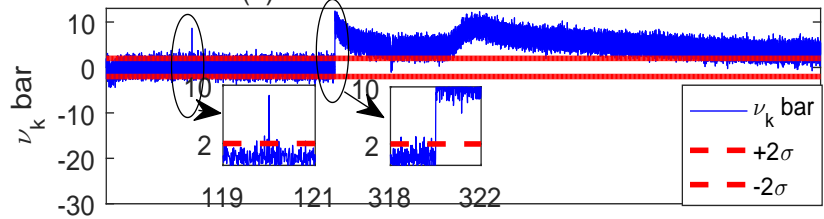

(c) Test1: Innovation magnitude test

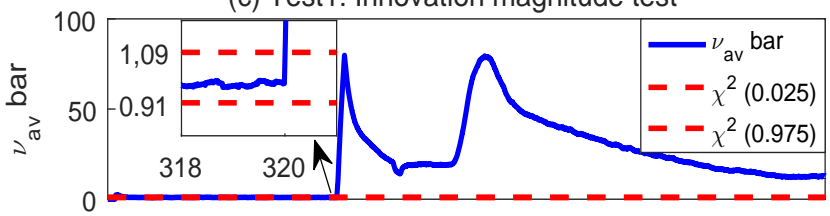

(d) Test2: Normalized innovation squared $\chi^{2}$ test

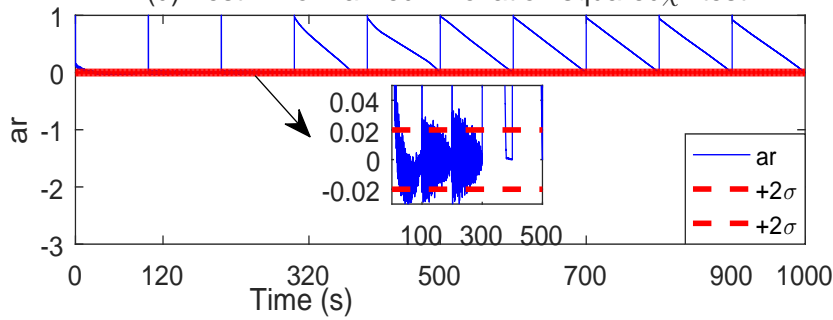

(e) Test3: Autocorrelation test of innovation

Fig. 14: Impact of bad data in $\Delta P_{m}$ on UKF performance and detection of bad data

\pm 3 was chosen, any normalized innovation sample beyond that zone may be considered as bad data and updating of states, covariance matrices are paused and a warning is issued.

\section{COnclusion}

This paper presents a method for accurate estimation of the parameters related to primary frequency control namely governor droop and dead-band. As the method relies on ambient data of continuous system load changes, it may be applied online and does not require data from large disturbances which are rare. The UKF was used for estimation as the 
system is non-linear because of the presence of governor deadband and UKF can handle non-linearity efficiently with small computational burden.

The performance of the method was tested for generators with GRC, and found to be satisfactory. It has also been shown that the method is robust to single outlier bad data in inputs $\Delta f$ and $\int A C E$ and also output $\Delta P_{m}$. In case of any outlier bad data in $\Delta P_{m}$, it can be detected from innovation magnitude test and hence estimation may be paused and updating of states and covariance matrices is also paused. However, the accuracy of the method is affected in presence of any permanent bad data or steady state error in measurements, but the bad data can be detected with three tests on innovation.

Continuous parameter estimation will help setting the frequency bias in secondary frequency control and optimizing primary frequency reserve.

\section{APPENDIX A}

TEST SYSTEM DATA

\begin{tabular}{|c|c|}
\hline$K_{p}=120 \mathrm{~Hz} / \mathrm{puMW}$ & $T_{p}=20 \mathrm{~s}$ \\
\hline$R=2.4 \mathrm{~Hz} / \mathrm{puMW}$ & $T_{t}=0.3 \mathrm{~s}$ \\
\hline$B=0.425 \mathrm{puMW} / \mathrm{Hz}$ & $T_{r}=10 \mathrm{~s}$ \\
\hline$d=0.015 \mathrm{puMW}$ & $F_{h p}=0.5$ \\
\hline $2 \times \pi \times T 12=0.545 \mathrm{puMW}$ & $T_{g}=0.08 \mathrm{~s}$ \\
\hline$p f_{i}=1$ & $K_{i}=0.05$ \\
\hline AGC Delay=5s & - \\
\hline
\end{tabular}

\section{REFERENCES}

[1] M. P. Roy Boyer et. al, Dynamic Models for Turbine-Governors in Power System Studies, IEEE Technical Report, 2013.

[2] G. Kou, P. Markham, S. Hadley, T. King, and Y. Liu, "Impact of Governor Deadband on Frequency Response of the U.S. Eastern Interconnection," IEEE Trans. Smart Grid, vol. 7, no. 3, pp.1368-1377,2016.

[3] P. Du, and Y. Makarov, "Using Disturbance Data to Monitor Primary Frequency Response for Power System Interconnections," IEEE Trans. Power Syst., vol. 29, no. 3, pp. 1431-1432, May 2014.

[4] J. Talaq, and F. Al-Basri, "Adaptive fuzzy gain scheduling for load frequency control, IEEE Trans. Power Syst., vol. 14, no. 1, pp. 145-150, 1999.

[5] "Policy 1 Generation Control and Performance," 2000.

[6] T. Kennedy, S. M. Hoyt, and C. F. Abell, "Variable, nonlinear tie-line frequency bias for interconnected systems control," IEEE Trans. Power Syst., vol. 3, no. 3, pp. 1244-1253, Aug. 1988.

[7] L.-R. Chang-Chien, N.-B. Hoonchareon, C.-M. Ong, and R. A. Kramer, "Estimation of for adaptive frequency bias setting in load frequency control,'IEEE Trans. Power Syst., vol. 18, no. 2, pp. 904-911, 2003.

[8] L.-R. Chang-Chien, Y.-S. Wu, and J.-S. Cheng, "Online estimation of system parameters for artificial intelligence applications to load frequency control," IET Gener. Transm. Distrib., Vol. 5, No. 8, pp. 895-902, 2011

[9] L. R. Chang-Chien, Y. J. Lin, and C. C. Wu, "A real-time contingency reserve scheduling for an isolated power system," IEEE Trans. Reliab., vol. 56, no. 1, pp. 139-147, 2007.

[10] L. R. Chang-Chien, Y. J. Lin, and C. C. Wu, "An online approach to allocate operating reserve for an isolated power system," IEEE Trans. Power Syst., vol. 22, no. 3, pp. 1314-1321, 2007.

[11] D. Jones, "Estimation of power system parameters,"IEEE Trans. Power Syst., vol. 19, no. 4, pp. 1980-1989, 2004.

[12] P. Kundur, Power System Stability and Control, year 2006.

[13] M. A. M. Ariff, B. C. Pal, and A. K. Singh,"Estimating Dynamic Model Parameters for Adaptive Protection and Control in Power System," IEEE Trans. on Power Syst., Vol. 30, No. 2, pp. 829-839, 2015.

[14] N. Zhou, J. W. Pierre, D. J. Trudnowski, and R. T. Guttromson,"Robust RLS methods for online estimation of power system electromechanical modes," IEEE Trans. Power Syst., vol. 22, no. 3, pp. 1240-1249, 2007.

[15] M. Yazdanian, A. Mehrizi-Sani, and M. Mojiri,"Estimation of Electromechanical Oscillation Parameters Using an Extended Kalman Filter,'IEEE Trans. Power Syst., Vol. 30, No. 6, pp. 2994-3002, 2015.
[16] K. Emami,et. al."Particle Filter Approach to Dynamic State Estimation of Generators in Power Systems,"IEEE Trans. Power Syst., vol. 30, no. 5, pp. 2665-2675, Sep. 2015.

[17] J. J. S. Gasca, C. J. Bridenbaugh, C. E. J. Bowler, and J. S. Edmonds, "Trajectory sensitivity based identification of synchronous generator and excitation system parameters,'IEEE Trans. Power Syst., vol. 3, no. 4, pp. 1814-1822, 1988.

[18] S. C. Tripathy, T. S. Bhatti, C. S. Jha, O. P. Malik, and G. S. Hope,"Sampled Data Automatic Generation Control Analysis with Reheat Steam Turbines and Governor Dead-Band Effects," IEEE Trans. Power Appar. Syst., vol. PAS-103, no. 5, pp. 1045-1051, May 1984.

[19] S. J. Julier and J. K. Uhlmann, "New extension of the Kalman filter to nonlinear systems," in Int Symp AerospaceDefense Sensing Simul and Controls, 1997, vol. 3, p. 182.

[20] (August, 2016) Load Data [Online] Available: http://www. nyiso.com/public/markets_operations/market_data/load_data/ index.jsp

[21] L. C. Saikia, S. Mishra, N. Sinha, and J. Nanda, "Automatic generation control of a multi area hydrothermal system using reinforced learning neural network controller," Int. J. Electr. Power Energy Syst., vol. 33, no. 4, pp. 1101-1108, 2011.

[22] T. Sezi,"A new method for measuring power system frequency,"in 1999 IEEE Trans. and Distrib. Conf., pp. 400-405, vol. 1, 1999.

[23] M. Karimi-Ghartemani and M. . Iravani,"Wide-range, fast and robust estimation of power system frequency," Electr. Power Syst. Res., vol. 65 , no. 2, pp.109-117, 2003.

[24] C. Hajiyev and H. E. Soken, "Robust adaptive unscented Kalman filter for attitude estimation of pico satellites," Int. J. Adapt. Control Signal Process., vol. 28, no. 2, pp. 107-120, 2014.

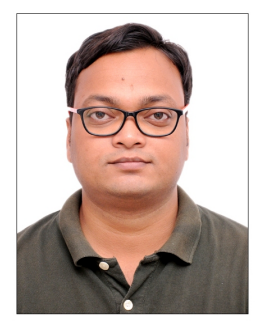

Pratyasa Bhui (S'16) received the M.Tech degree in power and energy system from the Indian Institute of Technology Kharagapur, Kharagpur, India, in 2013 and Ph.D. degree from the Indian Institute of Technology Delhi, New Delhi, India in 2017. His research interests include wide area monitoring, power system dynamics and grid integration of renewable energy.

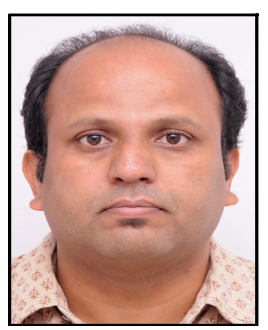

Nilanjan Senroy(M'16) received the M.S. and Ph.D. degrees from Arizona State University, Tempe, AZ, USA. He also has postdoctoral experience at the Center for Advanced Power Systems, Florida State University, Tallahassee, FL, USA. He is an Associate Professor in the Department of Electrical Engineering, Indian Institute of Technology, Delhi, India. His research interests include power system stability and control, signal processing, and renewable energy.

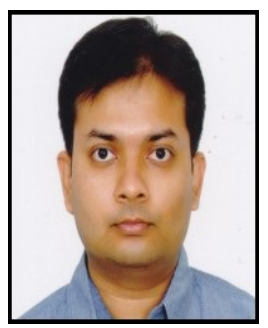

Abhinav K. Singh (S'12-M'15) received the B.Tech. degree from the Indian Institute of Technology, New Delhi, India, and the Ph.D. degree from Imperial College London, London, U.K., in 2010 and 2015, respectively, both in electrical engineering. Currently, he is working as a Lecturer of Electrical Engineering at University of Lincoln, UK, and as a visiting Research Fellow of Imperial College London. His current research interests include state estimation, networked control, and communication aspects of power systems.

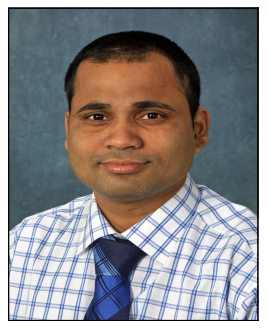

Bikash C. Pal (M'00-SM'02-F'13) received the B.E.E. degree from Jadavpur University, Kolkata, India, the M.E. degree from the Indian Institute of Science, Bangalore, India, and the Ph.D. degree from Imperial College London, London, U.K., in 1990, 1992, and 1999, respectively, all in electrical engineering. Currently, he is a Professor in the Department of Electrical and Electronic Engineering, Imperial College London. He is the Editor-in-Chief of IEEE TRANSACTIONS ON SUSTAINABLE ENERGY and Fellow of IEEE for his contribution to power system stability and control. His current research interests include state estimation and power system dynamics. 